\title{
Improvement of Obstacle and Singularity Avoidance Path Planning Algorithm for Redundant Manipulators
}

\author{
Liu Yu-Bin ${ }^{1, a}$, Dai Qian ${ }^{1, b}$ \\ ${ }^{1}$ Robotics Institute, Harbin Institute of Technology, Harbin 150001,China; \\ anature9966@163.com, bdaiqiansh@outlook.com
}

Keywords: Redundant manipulator, Obstacle avoidance, Singularity avoidance, Motion Planning

\begin{abstract}
A collaborative optimization scheme of obstacle avoidance and singularity avoidance path planning method is presented for redundant robot. An improved real-time minimum distance calculated method is presented and search the connect rod which easy to collision based on this minimum distance. Complete the obstacle avoidance based on the self-motion of the redundant manipulator on a null space and two obstacle avoidance parameters related to real-time minimum distance are introduced to improve optimization of obstacle avoidance. Adopt the DLS method to solve the problem that very high joint velocities in the vicinity of singular configuration. At last, through simulation of planar 3R redundant manipulator, the algorithm proves to be feasible and effective.
\end{abstract}

\section{Introduction}

The assembling work is more complicated as the miniaturization of 3C products and then the high speed manipulator was introduced by manufactures as auxiliary equipment of the assemble work to improve the assembly efficient and quality ${ }^{[1]}$. The risk of man-machine collaboration is increased significantly because the collaborators and the robot working space overlap in large areas while assembling product in man-machine collaboration ${ }^{[2]}$.

Moreover, the usual inverse kinematics solutions based on Jacobian pseudo-inverse cause very high joint velocities in the vicinity of singular configurations, and it will lead to the manipulator deviate from expected trajectory and will have a certain influence on assembly quality. So if we can design a path planning method which can make the robot complete obstacle and singularity avoidance automatically, then it will make the man-machine collaboration system large-scale application in the field of 3C product assemble ${ }^{[3]}$.

Compared with the traditional manipulator the redundant manipulator has the additional degrees of freedom, makes it has the advantages of flexible operation and can complete obstacle and singularity avoidance. In the field of obstacle-avoiding of the manipulator, the main research method including the artificial potential field method ${ }^{[4]}$,fuzzy method ${ }^{[5]}$, neural network method ${ }^{[6]}$, genetic algorithm ${ }^{[7]}$, Probabilistic Roadmaps method ${ }^{[8]}$,Rapidly-exploring Random Tree method ${ }^{[9]}$.However, these methods have some shortcomings and the contradiction between the optimal path the planning time and the complexity of algorithm is very difficult to solve. In the area of singularity-avoiding of the manipulator, the main research methods such as the Damped least square method ${ }^{[10]}$, SICQP method $^{[11]}$, normal forms method ${ }^{[12]}$. But these method is difficult to solve the contradiction of the tracking accuracy and the complexity of algorithm ${ }^{[13]}$.

An improved calculation method is proposed in this paper to compute the minimum distance in order to improve the real-time performance of the system. Two obstacle avoidance parameters related to minimum distance are introduced and the self-motion in null space of the redundant manipulator is utilized to accomplish obstacle avoidance. The Damped Least Square method is adopted to optimize manipulator performance in the vicinity of singular configurations, complete the optimization between singularity avoidance and obstacle avoidance for redundant manipulator. At last through simulation of 3-DOF redundant manipulator, the scheme proves to be feasible and effective. 


\section{Traditional distance calculation method}

For the traditional minimum distance calculation method, the first step is calculating the minimum distance between obstacles and each manipulator connecting bar of the manipulator and then taking the minimum value. Take a 3-DOF, for example, as shown in Figure 1(a), the real-time minimum distance can be described as $\mathrm{d}_{\min }=\min \left\{\mathrm{d}_{1}, \mathrm{~d}_{2}, \mathrm{~d}_{3}\right\}$.

While the manipulator motion to some special position such as shown in Figure 1(b), the pedal of the obstacle to the manipulator connecting bar may fell on the extension of the bar, because the extension of the rod is not part of the manipulator, so it need to selected some mark point from the connecting rod and compute the distance between the mark point and the obstacle, and then taking the minimum value. But this minimum distance is not accurate, and this method will lead to huge calculation, low efficiency and longtime of computation.

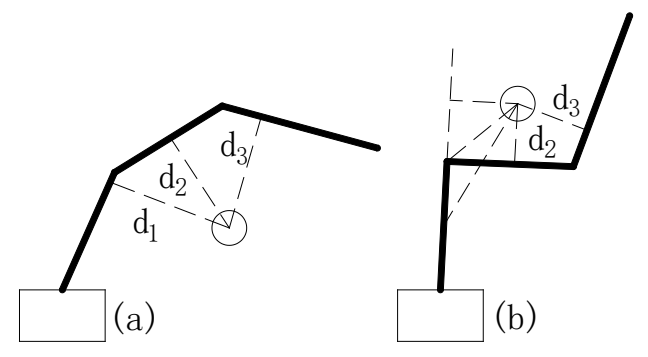

Fig.1 Traditional distance index

In order to solve this problem, a new method is proposed to calculate the minimum distance. The idea of the method is that the Cartesian coordinate established in the end of each connecting rod, and get the obstacle position in the each coordinate. Based on this, security rod that the pedal of the obstacle and the manipulator connecting bar fell on the extension of the road can be remove. That can reduce unnecessary distance calculation, improve the efficiency and accuracy of the algorithm.

\section{Improved distance calculation method}

As shown in Figure, the point $A$ is the origin of the coordinate system $\{0\}$ and $\{1\}$. The $X_{1}$ axis pointed in the direction of the connecting rod1 to connecting rod 2 and the $Y_{1}$ axis was perpendicular to the connecting rod 1 . Similarly, the origin of the coordinate system $\{2\}$ is fixed at B, the origin of the coordinate system $\{3\}$ fixed at the point $C$, the axis $X$ was all along the connecting rod and the axis $\mathrm{Y}$ was perpendicular to the connecting.

Take the link1, for example, determining whether it is safe link and calculating the minimum distance between it and the obstacle.

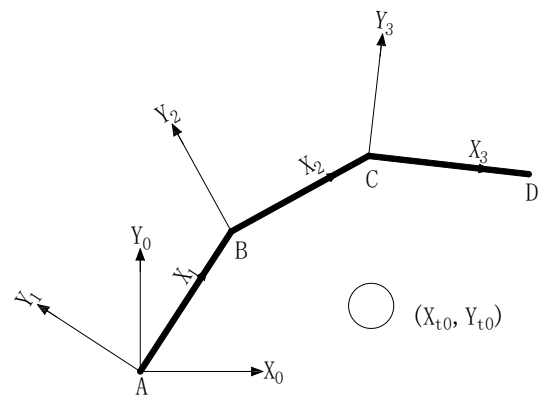

Fig.2 Position relation between the coordinates

The position of the obstacle center in the coordinate system $\{0\}$ can be get through some methods of measuring, and the connecting rod cross was a square which side length is a and the radius of the obstacle is $\mathrm{R}$.

Through the rotation matrix that the coordinate system $\{0\}$ relative to the coordinate system $\{1\}$, we can get the obstacle position $\left(\mathrm{x}_{\mathrm{t} 1}, \mathrm{y}_{\mathrm{t} 1}\right)$ in the coordinate system $\{1\}$, that can be written as 


$$
\left[\begin{array}{c}
x_{t 1} \\
y_{t 1} \\
0
\end{array}\right]=\left[\begin{array}{ccc}
\cos \theta_{1} & -\sin \theta_{1} & 0 \\
\sin \theta_{1} & \cos \theta_{1} & 0 \\
0 & 0 & 1
\end{array}\right]\left[\begin{array}{c}
x_{t 0} \\
y_{t 0} \\
0
\end{array}\right]
$$

If $\left(\mathrm{x}_{\mathrm{t} 1}, \mathrm{y}_{\mathrm{t} 1}\right)$ satisfied

$$
\left\{\begin{array}{c}
\left|y_{A 1}-y_{t 1}\right| \geq R+a \\
x_{A 1}-R \geq x_{t 1} \text { or } x_{t 1} \geq x_{B 1}+R
\end{array}\right.
$$

The link 1 is the safe rod, the distance between the obstacle and the rod don't need to calculate. If $\left(\mathrm{x}_{\mathrm{t} 1}, \mathrm{y}_{\mathrm{t} 1}\right)$ satisfied

$$
\left\{\begin{array}{c}
\left|y_{A 1}-y_{t 1}\right| \leq R+a \\
x_{A 1}-R \leq x_{t 1} \leq x_{B 1}+R
\end{array}\right.
$$

Then the link 1 may be collide with the obstacle, defined the mark point which was nearest closest to the obstacle in the rod. The position of the mark point $S$ in the coordinate system $\{1\}$ was $\left(x_{t 1}, 0\right)$ and the distance between the mark point $S$ and the obstacle was

$$
d_{1}=\left|y_{A 1}-y_{t 1}\right|
$$

Analyze the rod2 and rod3 according to the method mentioned before, calculated the distance between the obstacle and the mark point at the rod and take the minimum value to get the real-time minimum distance. Traditional minimum distance calculation was completed in the base coordinate system generally, the position of the obstacle was $\left(x_{t}, y_{t}\right)$, and the link AB equation can be written as

$$
\frac{\left(x-x_{A}\right)}{\left(x_{B}-x_{A}\right)}=\frac{\left(y-y_{a}\right)}{\left(y_{B}-y_{A}\right)}
$$

Perpendicular to the rod1 and passed through the center point of the straight line equation was

$$
\frac{\left(y-y_{a}\right)}{\left(x_{B}-x_{A}\right)}=\frac{\left(x_{A}-x\right)}{\left(y_{B}-y_{A}\right)}
$$

The position of the collision point can be get based on the simultaneous solution of two equations and then the distance can be calculated.

$$
d_{1}=\sqrt{\left(x_{S}-x_{t}\right)^{2}+\left(y_{S}-y_{t}\right)^{2}}
$$

Analyze the rod2 and rod3 according to the method state above, the minimum distance is $\mathrm{d}_{\min }=\min \left\{\mathrm{d}_{1}, \mathrm{~d}_{2}, \mathrm{~d}_{3}\right\}$.

Based on the above analysis, the improve distance calculated method which applied to completed the dynamic obstacle avoidance of redundant manipulator and improved the efficiency of the algorithm. This method meet the requirement of dynamic obstacle avoidance for real-time better, mainly reflected in two aspects:

1. Screened out the rod which may be collided with the obstacle before calculated the distance, and only counted the distance between this rods and the obstacle, that can reduce the amount of computation greatly.

2. The coordinate transformation is used to calculate the distance in the improved distance calculate method, abandoned the complicated computation of the solution equation in the traditional method that will reduce the amount of computation and improve the computation efficiency.

\section{Path planning of redundant manipulator}

The work space of the man and the manipulator overlap, then the likelihood of collision will be increased greatly while the man-machine collaboration to complete product assembly tasks. Moreover, the joint velocities will be very high even exceed the joint velocity limit, when the manipulator motion to the vicinity of singular configurations that will not only affect the quality of product assembly work and caused great security risk to collaborator. Obstacle and singularity 
avoidance path planning method can accomplish the obstacle avoidance and avoid the singular position at the same time, that can make the quality of assemble better and improved the man-machine cooperation security.

According to the method mentioned before, selected the rod may be collide with the obstacle and find the location of the mark point $S$ on the rod and the Jacobian in the point $S$ satisfied

$$
J_{s} \dot{q}=\dot{x}_{s}
$$

According to the gradient projection method, the redundant manipulator inverse solution is

$$
\dot{q}=J^{+} \dot{p}+\left(I-J^{+} J\right) H
$$

The obstacle avoidance function in null space can be written as

$$
H=\left[J_{0}\left(\left(I-J^{+} J\right)\right)\right]^{+}\left(\dot{x}_{s}-J_{S} J^{+} \dot{x}\right)
$$

Then the obstacle avoidance inverse kinematics of redundant manipulator based on the gradient projection method can be described as

$$
\dot{q}=J^{+} \dot{x}+\left(I-J^{+} J\right)\left[J_{S}\left(\left(I-J^{+} J\right)\right)\right]^{+}\left(\dot{x}_{s}-J_{S} J^{+} \dot{x}\right)
$$

Where $\dot{q} \in R^{n \times 1}$ is the joint velocity vector, $\dot{x} \in R^{m \times 1}$ is the end of the manipulator velocity vector, $J \in R^{m \times n}$ is the Jacobian matrix of the manipulator, $J_{S} \in R^{m \times n}$ is the Jacobian matrix in the mark point $\mathrm{S}$, $\dot{x}_{s}$ is the velocity vector of the mark point $S, J^{+} \in R^{n \times m}$ is Pseudo-inverse Jacobian。

Introduced the obstacle avoidance parameter a to replace $\left(I-J^{+} J\right)$ to simplify calculation, and in order to get a better effect of obstacle avoidance, introduced the obstacle avoidance parameter $b$, then the above equation can be described as

$$
\dot{q}=J^{+} \dot{x}+a\left[J_{s}\left(\left(I-J^{+} J\right)\right)\right]^{+}\left(b \dot{x}_{s}-J_{S} J^{+} \dot{x}\right)
$$

The obstacle avoidance parameter ' $a$ ' and ' $b$ ' were related to the real-time minimum distance, define two distance $\mathrm{d} 1$ and $\mathrm{d} 2$, they satisfied

$$
a=\left\{\begin{array}{ll}
A & d_{\text {min }}<d_{2} \\
0 & d_{\text {min }}<d_{2}
\end{array} \quad b= \begin{cases}B & d_{\text {min }}<d_{1} \\
0 & d_{\text {min }}>d_{1}\end{cases}\right.
$$

That is, while $\mathrm{d}_{\min }<\mathrm{d}_{1}$, the rod entered the dangerous zone the obstacle avoidance strategy started, while $\mathrm{d}_{\min }<\mathrm{d}_{2}$, the rod entered the extremely dangerous zone, the gain of obstacle avoidance started.

In the vicinity of the singular position configurations, the manipulator joint speed exceeded the limit easily, so the DLS method to optimize this problem was introduced.

First, take the singular value decomposition to the robot Jacobian matrix:

$$
J=A B C^{T}
$$

Where, A, C are orthogonal matrix, B is the diagonal matrix composed by the singular value of the manipulator Jacobian matrix.

$$
B=\left[\begin{array}{ccccccc}
\sigma_{1} & 0 & \cdot & \cdot & \cdot & \cdot & 0 \\
0 & \sigma_{2} & 0 & \cdot & \cdot & \cdot & 0 \\
\cdot & \cdot & \cdot & \cdot & \cdot & \cdot & \cdot \\
0 & \cdot & 0 & \sigma_{m} & 0 & \cdot & 0
\end{array}\right]
$$

Where, $\sigma_{1}, \sigma_{2}, \ldots, \sigma_{m}$ are the manipulator Jacobin matrix singular value and they satisfy that $\sigma_{1} \geq \sigma_{2} \geq \ldots \geq \sigma_{m}, \sigma_{m}$ is the minimum value of the Jacobian matrix, it can reflect the extent of nearly singular position of the manipulator.

Generally, Pseudo-inverse method was used to solve the inverse kinematics problem for redundant manipulator, but the essence of the method was solved the minimum norm joint velocity solution based on the end of the track deviation was minimized. In order to ensure minimize the tracking accuracy the Pseudo-inverse method ignored the impact of the joint velocity. Adopt the DLS method and the damping parameter $\mathrm{C}$ was introduced to regulated the priority of the tracking accuracy and joints velocity. The inverse kinematics solutions of the manipulator can be written as

$$
\dot{q}=J_{1} \dot{X}=J^{T}\left(J J^{T}+C^{2} I\right)^{-1} \dot{X}
$$


The tracking accuracy will decline while adopt the singularity robust inverse to solve the inverse kinematics problem, so the Pseudo-inverse method was used while the manipulator far away from the singular configuration and while the manipulator motion to the vicinity of singular configurations the singularity robust inverse was applied, then the damping parameter $C$ was be introduced

$$
C^{2}= \begin{cases}C_{0}^{2}\left(1-\frac{\sigma_{m}}{\sigma_{0}}\right) & \sigma_{m}<\sigma_{0} \\ 0 & \sigma_{m} \geq \sigma_{0}\end{cases}
$$

Where $C_{0}$ is the maximum damping parameter, $\sigma_{\mathrm{m}}$ is the minimum singular value, $\sigma_{0}$ is the lower limit of the singular values.

The manipulator motioned to the vicinity of singular configurations, that the singularity avoidance strategy started while $\sigma_{\mathrm{m}}<\sigma_{0}$. Used the Pseudo-inverse method to calculated the joint velocity while $\sigma_{\mathrm{m}}>\sigma_{0}$.

The singularity robust inverse was introduced to optimize the method of obstacle avoidance mentioned before, then the improved method can be described as

$$
\dot{q}=J_{1} \dot{x}+a\left[J_{0}\left(\left(I-J^{+} J\right)\right)\right]^{+}\left(b \dot{x}_{0}-J_{0} J^{+} \dot{x}\right)
$$

Where $J_{1}=J^{T}\left(J J^{T}+C^{2} I\right)^{-1}$

The flowchart of the obstacle and singularity avoidance path planning was shown in the following figure

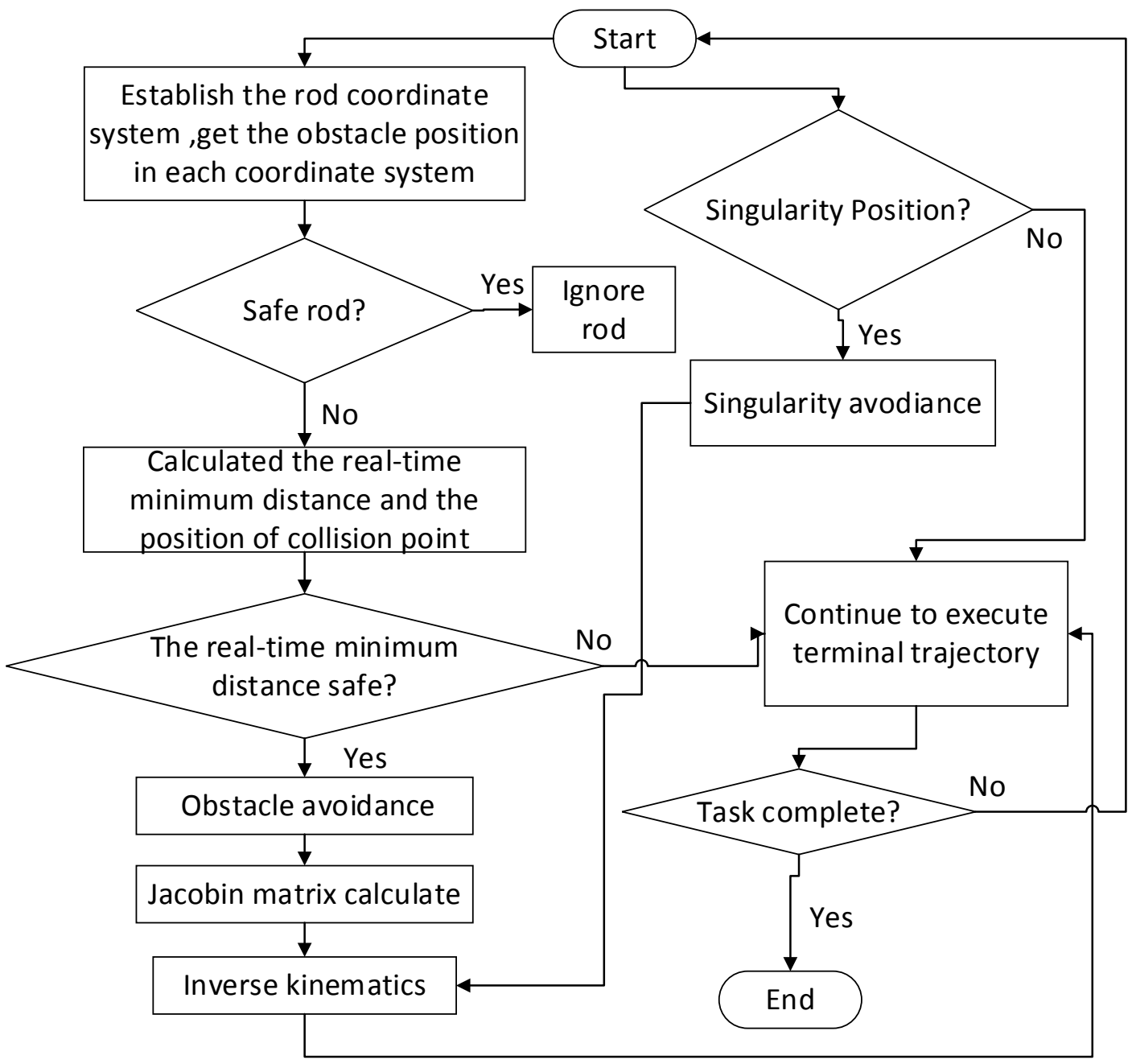

Fig.3 Flow chart of obstacle and singularity avoidance path planning

The obstacle and singularity avoidance path planning stated above realized the obstacle avoidance and singularity avoidance at the same times, and this algorithm features simplicity, less calculation requirement and the real-time performance of the system was further improved. 


\section{Simulation and Results Analysis}

Some simulation experiment to a 3-DOF redundant manipulator has been carried on the MATLAB2014 environment to prove the feasible of the scheme stated above. The D-H parameters was shown in the following table

Table 1 Robot D-H parameters

\begin{tabular}{ccccc}
\hline Rod & Variable & d & l & a \\
\hline 1 & $\theta_{1}$ & 0 & 200 & 0 \\
2 & $\theta_{2}$ & 0 & 200 & 0 \\
3 & $\theta_{3}$ & 0 & 200 & 0 \\
\hline
\end{tabular}

The end position of the plane 3-DOF redundant robot can be written as

$$
p=200\left[\begin{array}{l}
c_{12}+c_{1}+c_{123} \\
s_{12}+s_{1}+s_{123}
\end{array}\right]
$$

The Jacobian matrix of that is

$$
J=200\left[\begin{array}{ccc}
-s_{1}-s_{12}-s_{123} & -s_{12}-s_{123} & -s_{123} \\
c_{1}+c_{12}+c_{123} & c_{12}+c_{123} & c_{123}
\end{array}\right]
$$

where $s_{a b c}=\sin \left(\theta_{a}+\theta_{b}+\theta_{c}\right), \quad c_{a b c}=\cos \left(\theta_{a}+\theta_{b}+\theta_{c}\right)$

\section{Obstacle avoidance path planning.}

The initial joint angles of robot: $\theta_{1}=60^{\circ}, \theta_{2}=-60^{\circ}, \theta_{3}=-120^{\circ}$ there was a static obstacle with center at $(85,50)$ and radius was 40 in the manipulator's work space, the velocity of the end of the manipulator was $(0,-30)$, the simulation time was 5 s.

The curve of the minimum singular value was shown in the Fig.4, The manipulator wan not motioned to the vicinity of singular configurations, the singularity avoidance strategy was not started, Used the Pseudo-inverse method to calculated the joint velocity

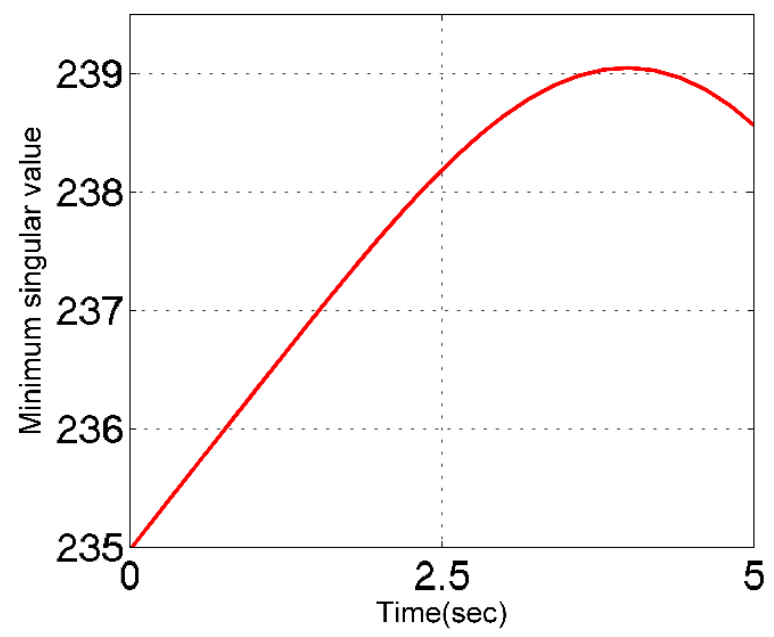

Fig.4 The minimum singular value curve

Contrasting fig. 5 and fig. 6 ,it is easy to find that the manipulator can avoid obstacles and track path accurately. 


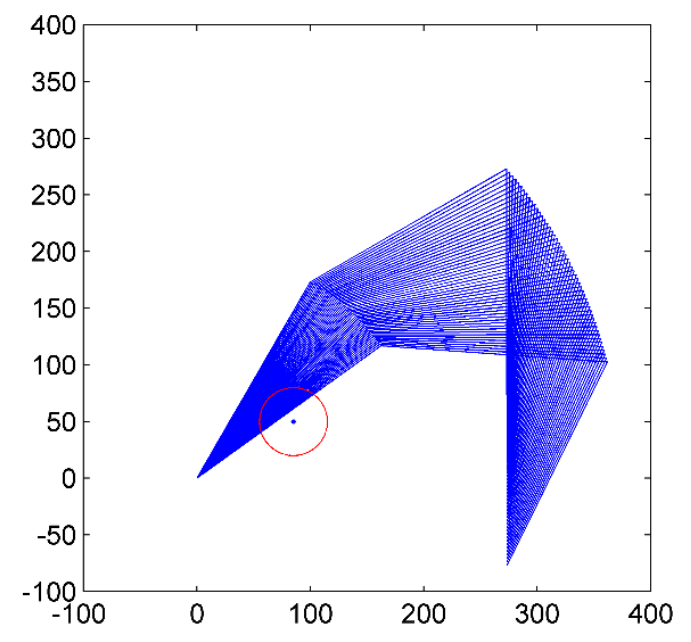

Fig.5 The movement locus before the optimization of obstacle avoidance

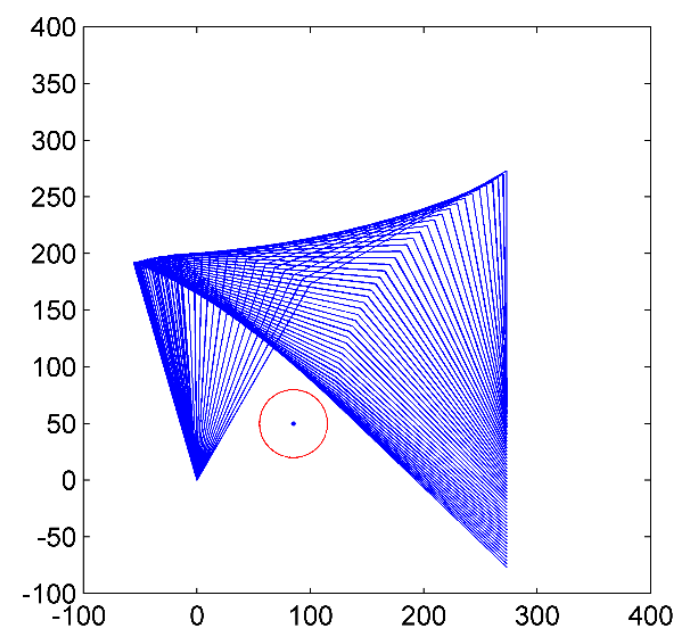

Fig.6 The movement locus after the optimization of obstacle avoidance

\section{Singularity avoidance path planning.}

The initial joint angles of robot: $\theta_{1}=60^{\circ}, \theta_{2}=-60^{\circ}, \theta_{3}=-45^{\circ}$,the velocity of the end of the manipulator was $(40,-60)$, the simulation time was $3.4 \mathrm{~s}$.

The curve of the minimum singular value was shown in the following figure, the minimum singular value equal to zero means that the manipulator motioned to the vicinity of singular configurations, the joint speed was very high in that time.

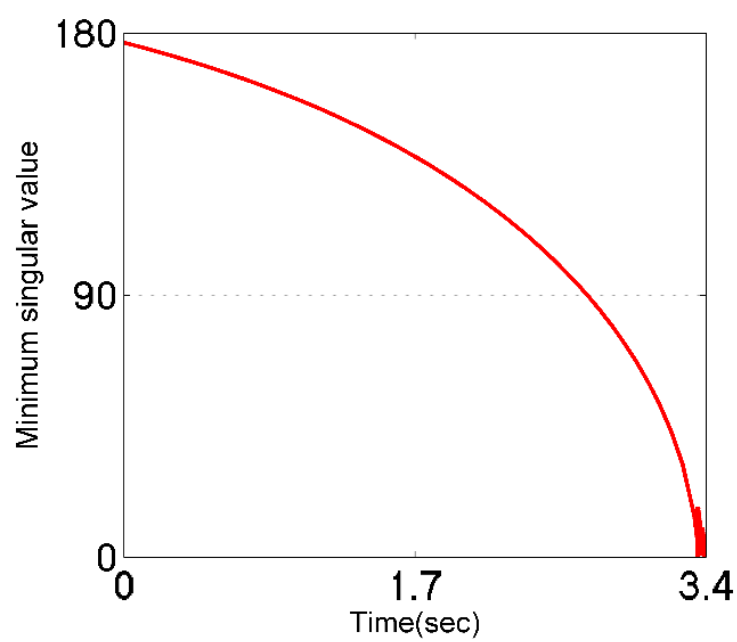

Fig.7 The minimum singular value curve before the optimization of singularity avoidance

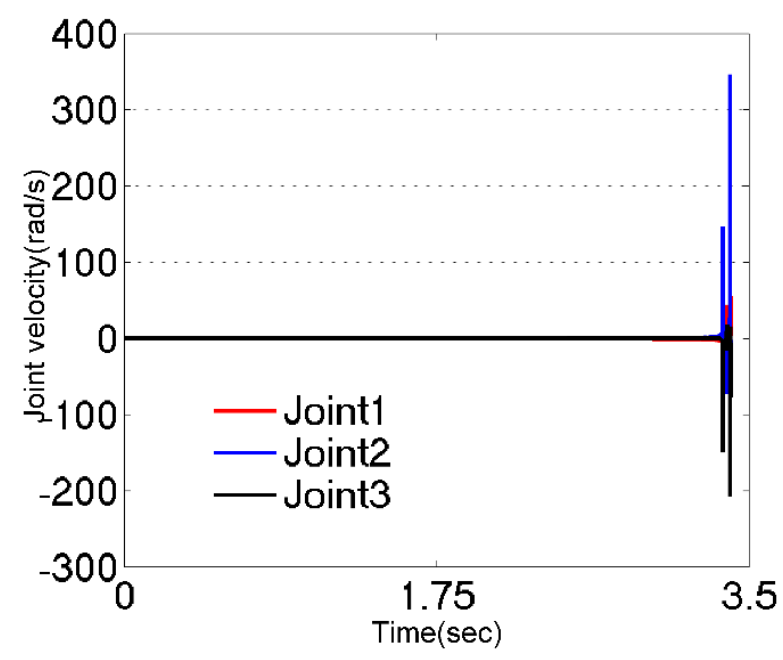

offset: 0

Fig.8 The joint angular velocity curve before the optimization of singularity avoidance 


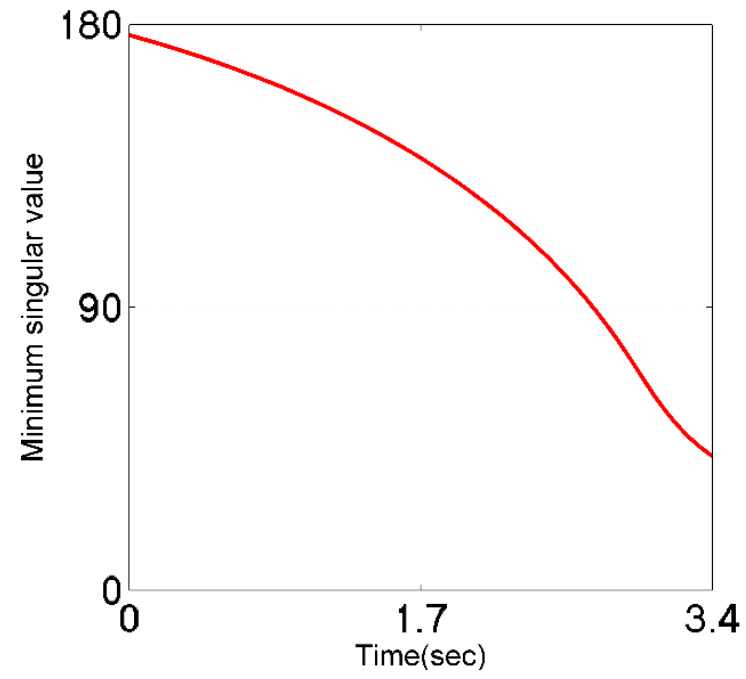

Fig.9 The minimum singular value curve after the optimization of singularity avoidance

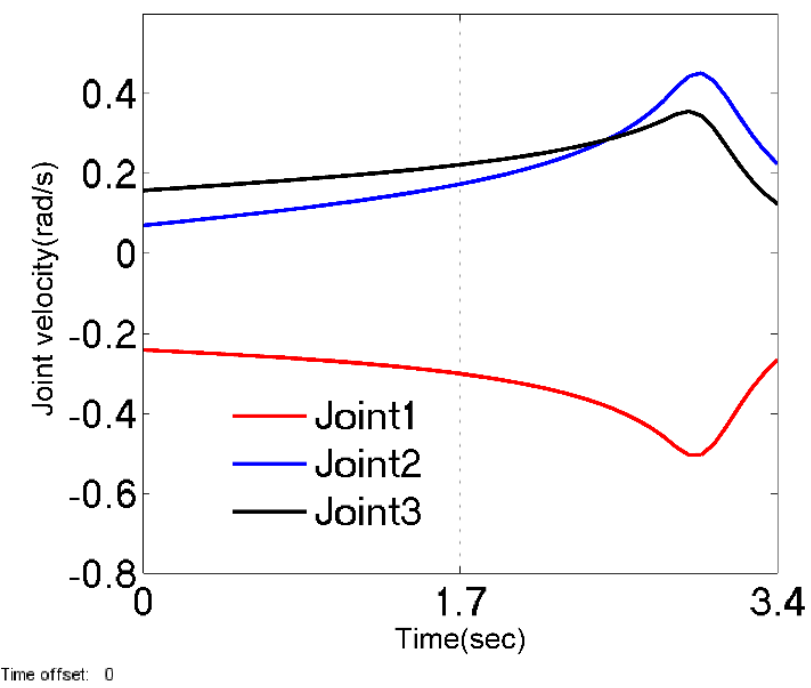

Fig.10 The joint angular velocity curve after the optimization of singularity avoidance

Compared fig7. with fig9. we can find that that the manipulator can avoid singularity and the motion was smooth enough.

After take the singularity avoidance strategy ,the simulation results are shown in Fig . There was no zero point at the curve of singular value, singular level was improved obviously compared with before optimization, and the motion was more stable than before

Obstacle avoidance path planning.

The initial joint angles of robot: $\theta_{1}=60^{\circ}, \theta_{2}=-60^{\circ}, \theta_{3}=-120^{\circ}$ there was a moving obstacle with center at $(85,50)$, radius was 40 and the speed was $(45,10)$ in the manipulator's work space, the velocity of the end of the manipulator was $(30,0)$, the simulation time was 7 s. The simulation results were the Fig.11-Fig.16.

Compared fig13. with fig16. we can find that that the manipulator can avoid singularity and obstacle at the same tines. 


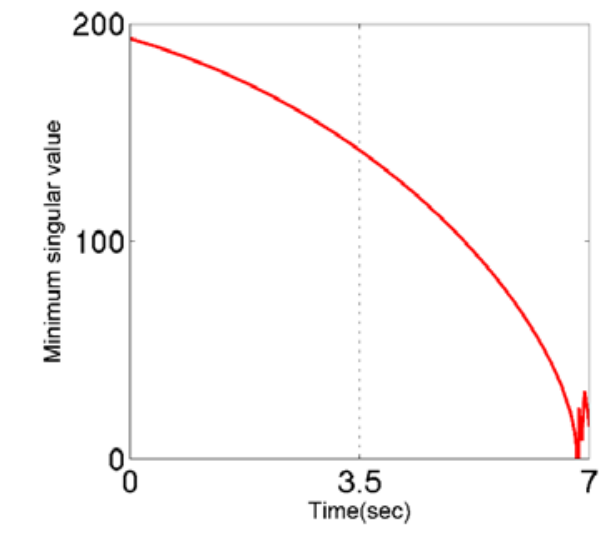

Fig.11 The minimum singular value curve after the optimization of obstacle and singularity avoidance

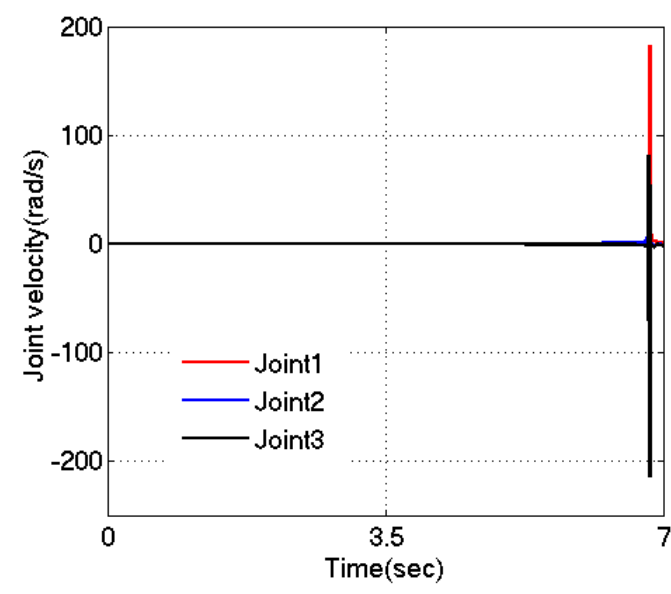

Time offset: 0

Fig.12 The joint angular velocity curve before the optimization of obstacle and singularity avoidance

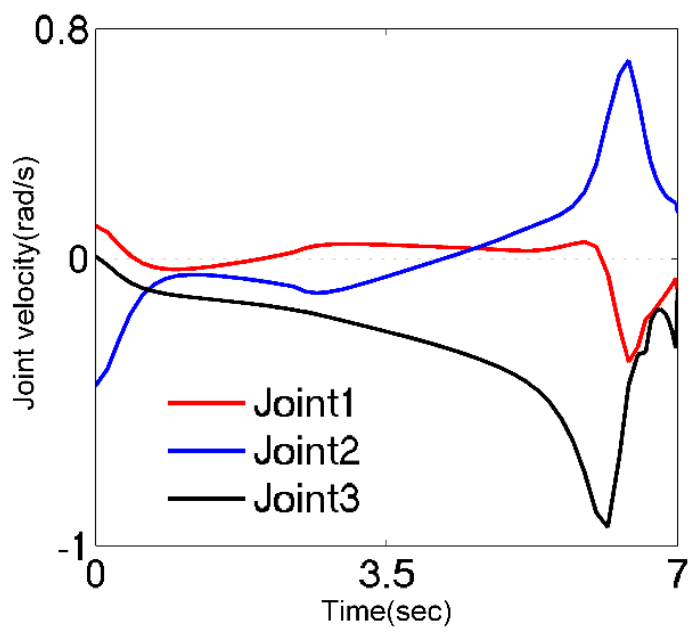

Fig.15 The joint angular velocity curve after the optimization of obstacle and singularity avoidance

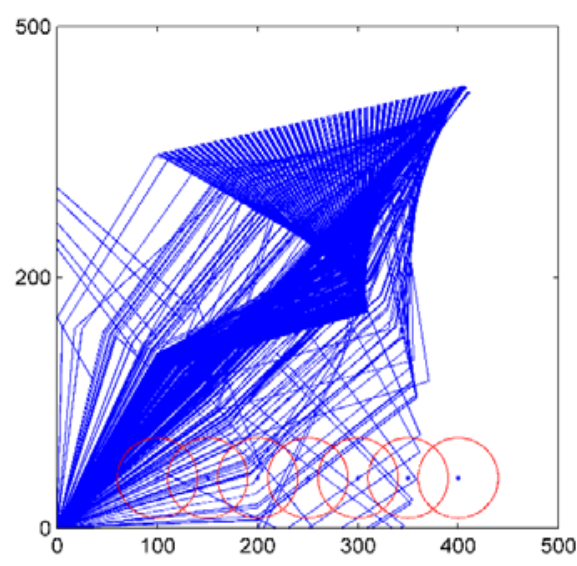

Fig.13 The movement locus before the optimization of obstacle and singularity avoidance

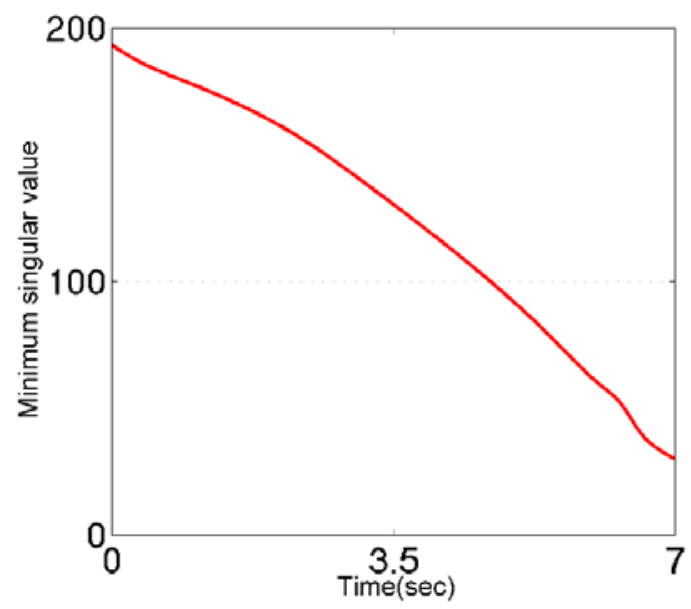

Fig.14 The minimum singular value curve after the optimization of obstacle and singularity avoidance

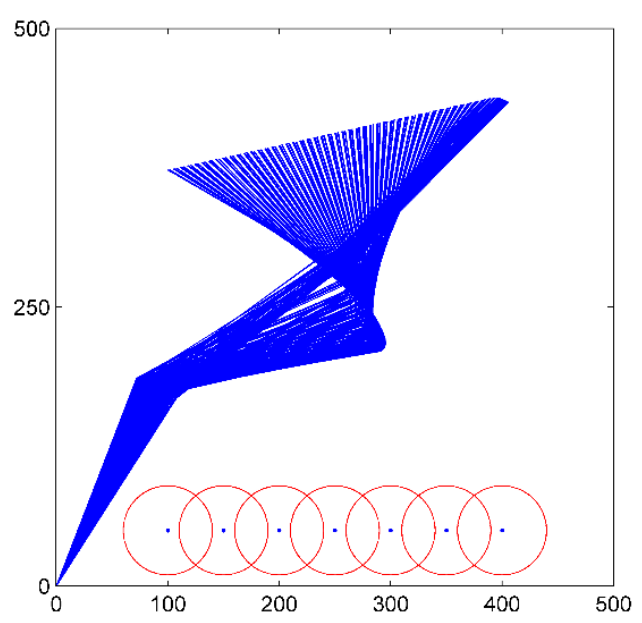

Fig.16 The movement locus after the optimization of obstacle and singularity avoidance 


\section{Summary}

How to completed obstacle and singularity avoidance was one of the key problem in human-machine collaboration process. In order to solve the problem of dynamic obstacle and singularity avoidance, this paper proposed an improved method to calculate the real-time minimum distance. Fulfilled the obstacle avoidance task through the self-motion of the redundancy kinematic chain and realized the singularity avoidance by used the DLS method. The results of the simulation experiment show that the path planning method proposed in this paper can make the manipulator completed the obstacle and singularity avoidance smoothly and steadily, still accomplish the trajectory tracking

\section{Acknowledgement}

This project has been supported by National High-tech R\&D Program of China (863 Program) 2015BAF10B00.

\section{References}

[1]. N. Phooripoom, P. Koomsap. Development of tiling automation for custom mosaic design[J]. Robotics and computer-integrated Manufacturing , v 35, p 55-68, October 1, 2015.

[2]. V. Grosu, C.R. Guerrero, B. Brackx, S. Grosu, B. Vanderborght, D. Lefeber. Instrumenting complex exoskeletons for improve human-robot interaction[J]. IEEE Instrumentation and Measurement Magazine, v 18, n 5, p 5-10, October 1,2015.

[3]. Y. Shen, G. Reinhart. Safe assembly motion-A novel approach for applying human-robot cooperation in hybrid assembly systems[C]. IEEE International Conference on Mechatronics and Automation, IEEE ICMA 2013, p 7-12, 2013.

[4]. H.T. Chiang, N. Malone, K. Lesser, M. Oishi, L. Tapia. Aggressive moving obstacle avoidance using a stochastic reachable set based potential field[J]. Springer Tracts in Advanced Robotics, v 107, p 73-89, 2015.

[5]. D. Davis, P. Supriya. Implementation of fuzzy-based robotic path planning[C]. Advances in Intelligent system and Computing, v 380, p 375-383, Proceeding of the 2nd International Conference on Computer and Communication Technologies, 2015.

[6]. W. Budiharto. Intelligent surveillance robot with obstacle avoidance capabilities using neural network[J]. Computational Intelligence and Neuroscience, v 2015, 2015.

[7]. N.M Rezk, Y. Alkabani, H. Bedor, S. Hammad. A distributed genetic algorithm for swarm robots obstacle avoidance[C]. IEEE International Conference on Computer Engineering and Systems, ICCES 2014, p 170-174, February 3, 2014.

[8]. M.T. Rantanen, M. Juhola. Using probabilistic roadmaps in changing environments[J]. Computer Animation and Virtual Worlds, v 25, n 1, p 17-31, January-February 2014.

[9]. J.L. Blanco, M. Bellone, A. Gimenez. TP-space RRT-Kinematic path planning of non-holonomic any-shape vehicles[J]. International Journal of Advanced Robotic Systems, v 12, may 13, 2015.

[10]. C. Bensalah, J. Gonzalez-Quijano, J.W. Zhang, M. Abderrahim. A new extended SDLS to deal with the JLA in the inverse kinematics of an anthropomorphic robotic hand[J]. Advances in Intelligent Systems and Computing, v 253, p 661-673, 2014.

[11]. H. Zhu, Y. Guan, M. Su, C. Cai, K.H. Low, H. Zhang. Evaluation of graspable region and selection of footholds for biped pole-climbing robots[C]. IEEE International Conference on Robotics and Biomimetics, IEEE ROBIO 2014, p 1757-1762, April 20, 2015. 
[12]. A. Veksler, T.A. Johansen, F. Borrelli, B. Realfsen. Cartesian thrust allocation algorithm with variable direction thrusters, turn rate limits and singularity avoidance[C]. IEEE Conference on Control Applications, CCA 2014, p 917-922, December 9, 2014.

[13]. C.W. Qiu, L.M. Wang, Y.W. Huang. Multi-objective planning of continuous trajectory task for an omni-directional mobile dual-arm robot based on product arbitration[J]. Robot, v 35, n 2, p 178-185, March 2013. 